\title{
Infrared high angular resolution measurements of stellar sources ${ }^{\star}$
}

\section{Accurate angular diameters of X Cnc, U Ori and Eta Gem}

\author{
A. Richichi ${ }^{1}$ and G. Calamai ${ }^{2}$ \\ 1 European Southern Observatory, Karl-Schwarzschildstr. 2, 85748 Garching bei München, Germany \\ 2 Osservatorio Astrofisico di Arcetri, L.go Enrico Fermi 5, 50125 Firenze, Italy
}

Received 25 October 2002 / Accepted 27 November 2002

\begin{abstract}
We present the results of near-IR lunar occultation measurements of three well-known, large angular diameter, cool giant stars, namely X Cnc (CII), U Ori (M8III) and $\eta$ Gem (M3III). Their $2.2 \mu \mathrm{m}$ angular diameters, derived under the assumption of a uniform circular disk, are $8.26 \pm 0.04,15.14 \pm 0.05$ and $12.57 \pm 0.04$ milliarcsec, respectively. In the case of $U$ Ori, we find a significant deviation of the brightness profile from circular symmetry, as well as a faint signature of extended emission. The asymmetry of the brightness profile cannot be explained by scintillation effects in the lunar occultation lightcurve, and could have contributed to the important differences in previous determinations of the angular diameter of U Ori in the near-IR. Also in the case of $\eta \mathrm{Gem}$, significant differences exist among the available angular diameter determinations.
\end{abstract}

Key words. techniques: high angular resolution - stars: fundamental parameters - stars: individual: X Cnc stars: individual: U Ori - stars: individual: Eta Gem

\section{Introduction}

This paper is the continuation of a series (Richichi \& Calamai 2001 and references therein) dedicated to angular diameter measurements of late-type stars obtained by the method of lunar occultations (LO). This program has been aimed mainly at the measurement of angular diameters of cool giants. The results have been used to obtain, in conjunction with the bolometric fluxes of the sources, the effective temperatures of $K$ and $M$ giants and carbon stars. An effective temperature calibration, derived in a homogeneous way using our LO measurements only, was presented by Richichi et al. (1999). Other stellar properties also have been measured for selected objects, such as limb-darkening, circumstellar shells and photospheric pulsation. Examples can be found in previous papers by our group, also outside the present series. Note that a similar series of papers has been published by our group devoted to LO observations of binary stars (Richichi et al. 1997 and references therein). LO results useful for the purpose of calibrating modern long-baseline interferometers have been included in the CHARM catalogue (Richichi \& Percheron 2002).

Send offprint requests to: A. Richichi,

e-mail: arichich@eso.org

* Based on observations collected at TIRGO (Gornergrat, Switzerland). TIRGO is operated by CNR - CAISMI Arcetri, Italy.
In the present paper we report on accurate measurements of three late-type giant stars, namely X Cnc (C6II), U Ori (M8III) and $\eta$ Gem (M3III). These stars stand out from the average of our diameter results because of their large angular sizes, which are of the order of 10 milliarcsec (mas). The high angular resolution enabled by LO has permitted us not only to determine their angular diameters, but also to investigate details of their brightness distribution and possible deviations from the simple model of a homogeneous disk. The method of observation and the data analysis are briefly summarized in Sect. 2, while more details can be found in the previous papers of this series. In Sect. 3 we present and discuss the results individually for each star. Because of their brightness and large angular sizes, these stars have been studied also by other high angular resolution methods and several previous determinations are available. A discussion in the context of previous measurements is also provided.

\section{Observations and data analysis}

Table 1 summarizes the details of the observations, following the format of previous papers in the series. In Cols. (1) and (2) we list the source name and the date of the LO event. Column (3) lists the code of the telescope used, which for the observations presented here was always the $1.5 \mathrm{~m}$ TIRGO telescope. In Cols. (4) through (7) we list the predicted position 
Table 1. Summary of the occultation observations.

\begin{tabular}{cccccccc}
\hline \hline$(1)$ & $(2)$ & $(3)$ & $(4)$ & $(5)$ & $(6)$ & $(7)$ & $(8)$ \\
Source & $\begin{array}{c}\text { Date } \\
\mathrm{UT}\end{array}$ & Tel. & $\begin{array}{c}\text { PA } \\
\circ\end{array}$ & $\begin{array}{c}D \\
\text { " }\end{array}$ & $\begin{array}{c}\Delta t \\
\mathrm{~ms}\end{array}$ & $\begin{array}{c}\tau \\
\mathrm{ms}\end{array}$ & $\begin{array}{c}\lambda \\
\mu \mathrm{m}\end{array}$ \\
\hline X Cnc & $28-11-99$ & $\mathrm{~T}$ & 229 & 28 & 1.92 & 1.50 & $\mathrm{~K}$ \\
U Ori & $13-03-00$ & $\mathrm{~T}$ & 75 & 28 & 1.42 & 1.01 & $\mathrm{~K}$ \\
$\eta$ Gem & $31-03-01$ & $\mathrm{~T}$ & 22 & 21 & 2.42 & 2.00 & $\mathrm{~K}$ \\
\hline
\end{tabular}

angle (PA) of occultation, the field of view of the photometer, the sampling time of the lightcurve and the integration time of each data point. The filter or wavelength of observation is reported in Col. (8).

All occultations were observed with the TIRGO facility fast photometer. Details can be found in Richichi et al. (1997), and references therein. All stars were observed with a standard broad-band $K$ filter. The same photometer was also used to obtain near-IR photometry close to the date of the LO events, when possible.

The analysis of the LO data was carried out, as in the other papers of this series, by means of a code based on the leastsquares method (LSM) and including corrections for biases due to the finite time response of the instrument, atmospheric scintillation, and pick-up frequencies. These features, as well as the details of the error estimation, have also been described in previous papers of the series. The LSM code is well suited for cases in which a model can be provided for the source, and can be characterized by a few parameters. For cases in which the source showed a complex structure, a model-independent approach was needed. For this, we have used the CAL method (Richichi 1989). This latter, starting from an initial featurless brightness profile, converges towards the most probable solution according to the maximum-likelihood definition, using the Lucy-Richardson deconvolution algorithm.

\section{Results and discussion}

Our angular diameter results are listed in Table 2. They have been obtained under the hypothesis of a uniform disk (UD). The difference from a limb-darkened (LD) diameter in the $K$ band for late-type non-Mira giants is predicted to be at the level of few percent (Hofmann \& Scholz 1998). Computations of limb-darkened LO diameters using a grid of numerical center-to-limb variation models were reported by Richichi et al. (1999), confirming this prediction. Even with the good signalto-noise ratio $(S N R)$ of the data sets discussed in this paper (see Table 2), a direct determination of the LD diameter is not very reliable. We have thus preferred to continue the general approach used in this series of papers, and have used UD diameters throughout.

\subsection{Cnc}

A LO lightcurve of good quality could obtained for this carbon star. After including the signal fluctuations due to scintillation at temporal frequencies lower than those of the diffraction fringes, the data could be well fitted by a single uniform
Table 2. Summary of results.

\begin{tabular}{lcrrc}
\hline \hline Source & Sp. & \multicolumn{1}{c}{$\phi_{\text {UD }}$} & SNR & Comments \\
\hline X Cnc & C6II & $8.26 \pm 0.04$ & 127 & \\
U Ori & M8III & $15.14 \pm 0.05$ & 300 & asymmetry, shell \\
$\eta$ Gem & M3III & $12.57 \pm 0.04$ & 58 & \\
\hline
\end{tabular}

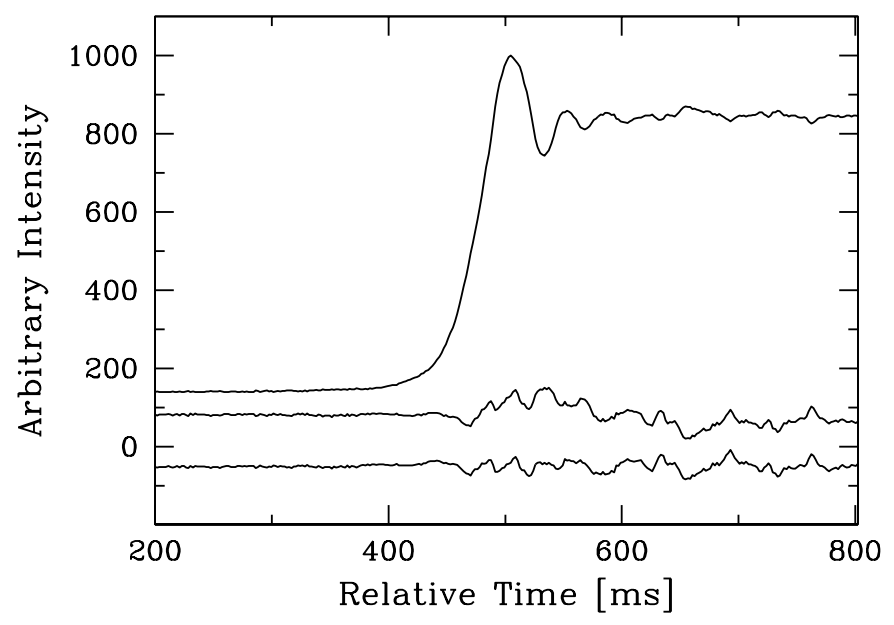

Fig. 1. From top to bottom: the occultation trace of X Cnc; the residuals of a fit by a uniform circular disk model, enlarged by a factor of two; same, after removing the effects of scintillation.

circular disk model, as shown in Fig. 1. Note that in the figures of this paper we do not show the actual fits. Given the good quality, they would be difficult to distinguish from the data. We have decided to show only the fit residuals.

Our derived value is $8.26 \pm 0.04$ mas. Two other measurements exist in the literature for X Cnc, both obtained by means of LO. Bartholdi et al. (1972) measured a uniform-disk diameter of $7.9 \pm 0.8$ mas at $0.69 \mu \mathrm{m}$. Ridgway et al. (1982) obtained under the same hypothesis a diameter of $7.72 \pm 0.16$ mas at $1.6 \mu \mathrm{m}$. The difference between our determination and this latter can probably be explained by wavelength effects in the stellar atmosphere, such as opacity. The variety of models available is sufficiently large, and their dependence on detailed assumptions in the atmospheric composition sufficiently marked (Johnson 1982), that it is difficult to pinpoint an exact quantitative expectation value for the changes in diameter with wavelength. Conversely, one might hope that accurate measurements at various wavelengths might provide sufficient input to constrain the models. Bergeat et al. (2001) have included X Cnc in their study of the effective temperatures of carbon stars, and derived a value of $2660 \pm 120 \mathrm{~K}$. With our measurement, their adopted value for the angular diameter would have to be corrected by about $5 \%$, implying a slightly lower temperature of $\approx 2600 \mathrm{~K}$. This is consistent with the error bars, and with the average effective temperature of the CV5 group to which X Cnc belongs in the classification by the above authors.

Photometry was obtained at the TIRGO telescope around the date of the LO, and the details are listed in Table 3. 
Table 3. TIRGO near-IR photometry of X Cnc.

\begin{tabular}{cccrr}
\hline \hline Date & \multicolumn{1}{c}{$J$} & \multicolumn{1}{c}{$H$} & \multicolumn{1}{c}{$L$} \\
\hline $26-11-99$ & $1.74 \pm 0.02$ & $0.79 \pm 0.02$ & $0.05 \pm 0.02$ & $-0.36 \pm 0.17$ \\
$15-01-00$ & $1.78 \pm 0.03$ & $0.68 \pm 0.04$ & $0.05 \pm 0.03$ & $-0.68 \pm 0.05$ \\
$16-01-00$ & $1.89 \pm 0.02$ & $0.80 \pm 0.02$ & $-0.09 \pm 0.02$ & $-0.34 \pm 0.05$ \\
$19-01-00$ & $1.83 \pm 0.02$ & $0.74 \pm 0.02$ & $-0.05 \pm 0.02$ & $-0.45 \pm 0.05$ \\
\hline
\end{tabular}

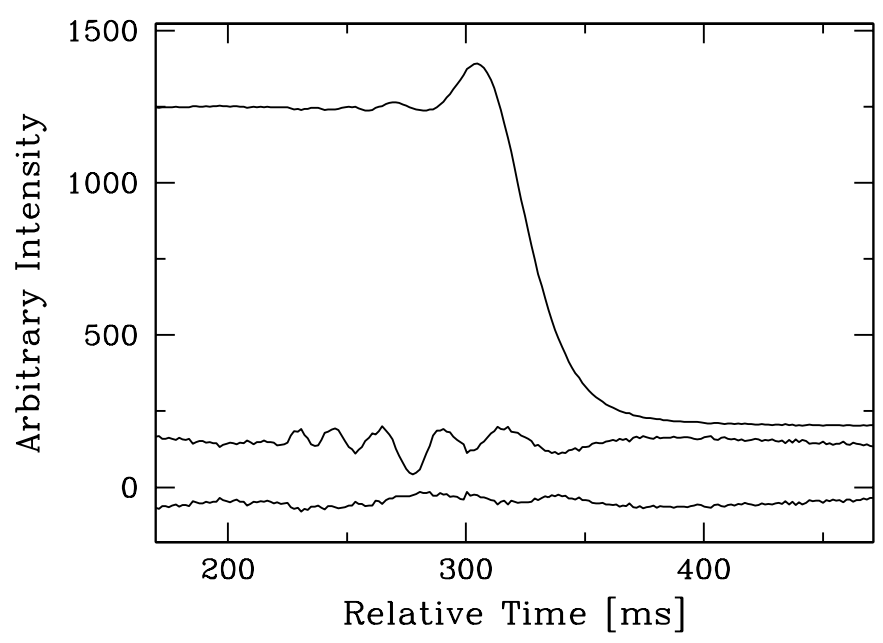

Fig. 2. From top to bottom: the occultation trace of U Ori; the residuals of a fit by a uniform circular disk model, enlarged by a factor of four; the residuals of a fit by the model-independent method CAL (see also Fig. 3), also enlarged by a factor of four.

\subsection{U Ori}

The angular diameter value of $15.14 \pm 0.05$ mas that we derive by a uniform disk fit is consistent with previous measurements by LO by Ridgway (1977), which resulted in $14.30 \pm 0.54$ mas and $15.40 \pm 0.33$ mas in the $H$ and $K$ band respectively. Two more determinations by LBI, also in the $K$ band, resulted in significantly smaller values: Van Belle et al. (1996) and Berger et al. (2001) obtained $10.86 \pm 0.56$ mas (LD diameter) and $11.0 \pm 0.5$ mas, respectively.

We note that measurements at wavelengths other than $H$ and $K$ yielded substantially larger values for the characteristic size of U Ori. In particular, Chagnon et al. (2002) measured U Ori by LBI in the $L^{\prime}$-band $(25.66 \pm 0.31$ mas), while Danchi et al. (1994) used the ISI heterodyne interferometer at $11.4 \mu \mathrm{m}$ (inner shell radius of 80 mas). These values are indicative of a significant, or even substantial, effect of thermal emission from the surrounding dust. Haniff et al. (1995) measured the angular diameter of $U$ Ori by the technique of aperture masking in the visible range, and also obtained values larger than the nearIR ones, from 18.5 to 24.7 mas depending on the filter used and on the adopted model. For these observations, the difference is probably explained as effects of varying optical depth, to which the narrow filters used by the authors were particularly sensitive.

A close inspection of the residuals of our model-dependent fit, shown enlarged in Fig. 2, reveals however systematic fluctuations. A fit by the CAL method provided a much better agreement with the data, and resulted in the brightness profile shown

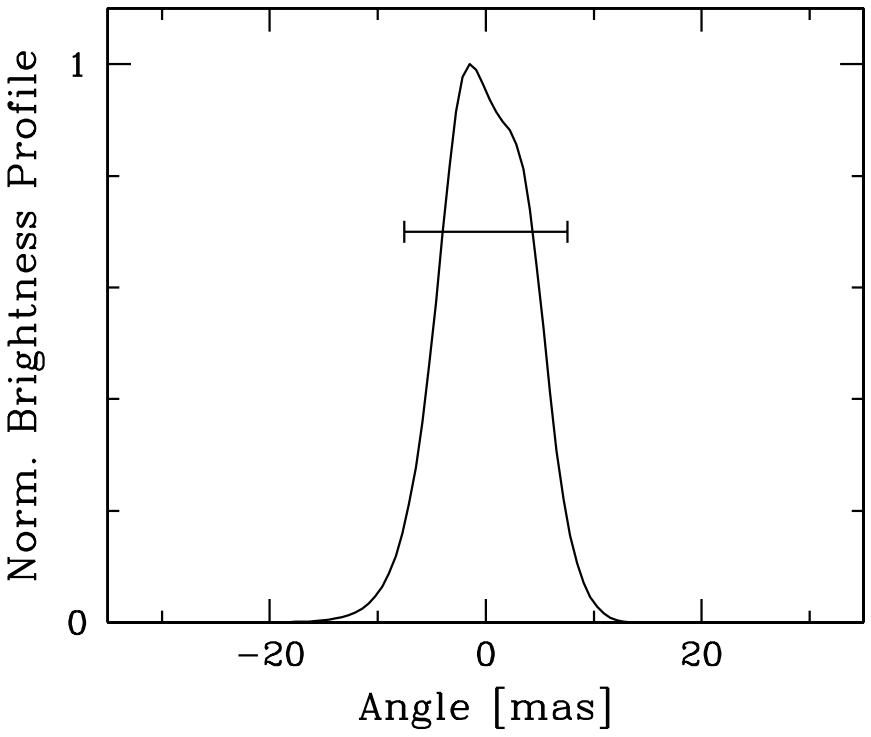

Fig. 3. Brightness profile of U Ori derived using a model-independent analysis. The horizontal segment marks the size of the equivalent uniform disk diameter (see Table 2).

in Fig. 3. The $S N R$ of the fit increased by almost a factor of two with respect to the model-dependent analysis, while the reduced $\chi^{2}$ decreased from 4.2 to 3.7. The fact that the reduced $\chi^{2}$ remained substantially larger than unity is due to residual systematics, mainly caused by scintillation. We did not attempt to remove scintillation effects in this case, since in general this could lead to contamination of the derived brightness profile. However, an inspection of Fig. 2 shows that the temporal frequency of scintillation is much slower than the errors due to the circular UD assumption. We conclude that the result shown in Fig. 3 is not biased by scintillation.

In addition to the inhomogeneities of the stellar surface evident from Fig. 2, the analysis also showed faint wings of the brightness profiles extending to about 20-30 mas, or 1-3 stellar radii. The signature of these features is too faint to derive quantitative measurements, but one could infer that they are due to extended circumstellar emission. The total flux in the extended component would appear to be $<1 \%$ of the stellar flux.

The picture that emerges is that of an object with a significant amount of circumstellar matter, probably very close to the stellar surface, as has been found in similar objects before. At wavelengths shorter than the near-IR, scattering is important and results in the large charactestic size found by Haniff et al. (1995). At wavelengths longward of the near-IR, thermal emission from the circumstellar matter becomes prominent and explains also the large diameters measured 3.5 and $11 \mu \mathrm{m}$. In the near-IR, we are probably detecting the photosphere. The fact that there is a significant difference between the angular diameter determinations in $H$ and $K$ obtained by LO and by LBI could be tentatively explained by the significant departure from the circular UD model, as shown in Fig. 3. The effect of such asymmetries is different on LO lightcurves and LBI visibilities, and furthermore one would have to consider the actual position angles of the various observations. The difference between the two groups of LO and LBI measurements amounts to $\approx 15$ mas 


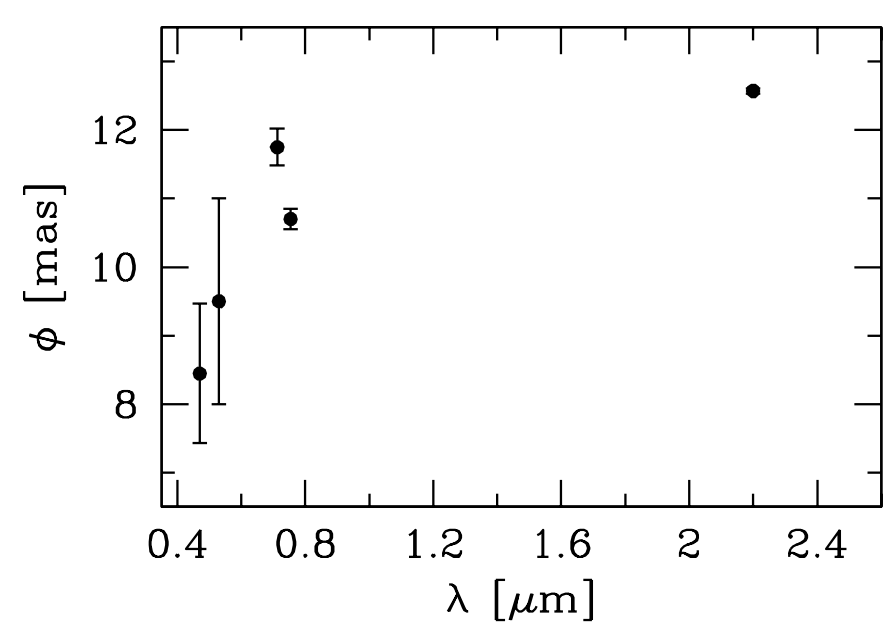

Fig. 4. Existing determinations of the angular diameter of $\eta \mathrm{Gem}$, including the result of the present work at $2.2 \mu \mathrm{m}$, as a function of wavelength. References to the other measurements are given in the text.

versus $\approx 11$ mas respectively, or about $5 \sigma$. It is sufficiently large to affect significantly the determination of the photospheric effective temperature. We note that, at the late M8 spectral type reported for for U Ori, also pulsation could play a role.

On the night of March 15, 2000 we also obtained nearIR photometry from TIRGO, which resulted in magnitudes of $0.87 \pm 0.10,-0.10 \pm 0.11,-0.88 \pm 0.03$ in the $J, H, K$ bands respectively.

\section{3. $\eta \mathrm{Gem}$}

A disapperance of $\eta$ Gem was recorded on March 31, 2001. Given that the event occurred at a large airmass $(z=1.75)$, the lightcurve is affected by some scintillation. Thanks also to a favorable contact angle of $-70^{\circ}$, which reduced the scan rate to about one half of the typical value, scintillation could be adequately sampled and accounted for in our fit. The final quality is sufficient for an accurate diameter measurement, resulting in a value of $12.57 \pm 0.04$ mas. At the level of the $S N R$ available for this measurement, a uniform disk model was found to adequately fit the data.

Two early LO measurements in the visual range had yielded slightly smaller determinations of the angular diameter of this M2.5 giant. In particular, Böhme (1978) and Schmidtke et al. (1984) obtained $9.5 \pm 1.5$ and $8.45 \pm 1.02$ mas respectively. Quirrenbach et al. (1993) used LBI to obtain measurements in a $\mathrm{TiO}$ absorption band and nearby continuum at 0.712 and $0.754 \mu \mathrm{m}$, obtaining $11.75 \pm 0.27$ and $10.70 \pm 0.15 \mathrm{mas}$ respectively. The discrepancy between all these measurements, including our own, is relatively small in absolute terms but reaches several sigmas. Figure 4 summarizes these results as a function of wavelength. Since no pulsational phenomena are known in the case of $\eta$ Gem, nor are they expected at this spectral type, further investigations are desirable to explain the differences, and a possible wavelength dependency.
No additional photometry could be obtained by us for this star around the time of the occultation.

\section{Conclusions}

We have presented lunar occultation data with high signal-tonoise ratio for three late-type giant stars, namely X Cnc (CII), U Ori (M8III) and $\eta$ Gem (M3III). Their angular diameters could be derived, with an accuracy significantly higher than other determinations available until now for these stars. While for X Cnc our result can be considered a refinement of previous determinations, in the case of $\mathrm{U}$ Ori and $\eta$ Gem significative differences exist, or already existed prior to this paper. In the case of U Ori, we find evidence of a non-symmetric brightness profile, as well as of a faint extended circumstellar emission. The asymmetry in the brightness profile, as well as possible pulsation phenomena in the atmosphere of this very cool giant star, could be the key to explain the differences among existing angular diameter determinations. The same explanation cannot be invoked in the case of $\eta \mathrm{Gem}$.

Acknowledgements. This research has made use of the Simbad database, operated at the Centre de Données Astronomiques de Strasbourg (CDS), and of NASA's Astrophysics Data System Bibliographic Services (ADS).

\section{References}

Bartholdi, P., Evans, D. S., \& Mitchell, R. I. 1972, AJ, 77, 756

Bergeat, J., Knapik, A., \& Rutily, B. 2001, A\&A, 369, 178

Berger, J. P., Haguenauer, P., \& Kern, P. 2001, A\&A, 376, L31

Böhme, D. 1978, AN, 299, 243

Chagnon, G., Mennesson, B., Perrin, G., et al. 2002, AJ, 124, 2821

Danchi, C. W., Bester, M., Degiacomi, C. G., Greenhill, L. J., \& Townes, C. H. 1994, AJ, 107, 1469

Haniff, C. A., Scholz, M., \& Tuthill, P. G. 1995, MNRAS, 276, 640

Hofmann, K.-H., \& Scholz, M. 1998, A\&A, 335, 637

Johnson, H. R. 1982, ApJ, 260, 254

Quirrenbach, A., Mozurkewich, D., Armstrong, J. T., Busher, D. F., \& Hummel, C. A. 1993, ApJ, 406, 215

Richichi, A. 1989, A\&A, 226, 366

Richichi, A., Calamai, G., Leinert, Ch., \& Stecklum, B. 1997, A\&A, 322, 202

Richichi, A., Fabbroni, L., Ragland, S., \& Scholz, M. 1999, A\&A, 344,511

Richichi, A., \& Calamai, G. 2001, A\&A, 380, 526

Richichi, A., Calamai, G., \& Stecklum, B. 2001, A\&A, 382, 178

Richichi, A., \& Percheron, I. 2002, A\&A, 386, 492

Ridgway, S. T. 1977, AJ, 82, 511

Ridgway, S. T., Jacoby, G. H., Joyce, R. R., Siegel, M. J., \& Wells, D. C. $1982, \mathrm{AJ}, 87,808$

Schmidtke, P. C., \& Africano, J. L. 1984, AJ, 89, 1371

Van Belle, G. T., Dyck, H. M., Benson, J. A., \& Lacasse, M. G. 1996, AJ, 112, 2147 